Working Paper 94-32

Statistics and Econometrics Series 13

September 1994
Departamento de Estadística y Econometría

Universidad Carlos III de Madrid

Calle Madrid, 126

28903 Getafe, Madrid (Spain)

Fax (341) 624-9849

\title{
A MULTIVARIATE KOLMOGOROV-SMIRNOV TEST OF GOODNESS OF FIT
}

\author{
Ana Justel Daniel Peña and Rubén Zamar ${ }^{1}$
}

\begin{abstract}
This paper presents a distribution free multivariate Kolmogorov-Smirnov goodness of fit test. The test uses an statistic which is built using Rosenblatt's transformation and an algorithm is developed to compute it in the bivariate case. An approximate test, that can be easily computed in any dimension, is also presented. The power of these multivariate tests is studied in a simulation study.
\end{abstract}

Key words:

Empirical distribution function. Kolmogorov-Smirnov statistics. Rosenblatt's transformation.

'Justel and Peña, Departament of Statistics and Econometrics, Universidad Carlos III de Madrid and Zamar, Department of Statistics, University of British Columbia. 


\section{INTRODUCTION}

Goodness of fit tests have been mostly developed for univariate distributions and, except for the case of multivariate normality, very few references can be found in the literature about multivariate goodness of fit tests. (See Krishnaiak, 1980, Kotz and Johnson, 1983 and D'Agostino and Stephens, 1986).

In principle, the cli-square test can be applied for testing any multivariate distribution but it is unknown what is the best way to choose the cell limits and what is the best statistic to be used. Moore and Stubblebine (1981) suggested choosing as cell boundaries the concentric hyperellipses centered at the sample mean and with shape determined by the inverse of the covariance matrix, and used the Rao and Robson (1974) modification of the chi-squared statistic. However, much work need to be done on the properties of this test.

The two most important classes of tests of goodness of fit based on the empirical distribution function of a random sample, the Kolmogorov-Smirnov statistic and the Cramer-von Mises group, have not been extended to the multivariate case. The problem is that the probability distribution of these multivariate statistics are not distribution free as in the univariate case. Rosenblatt (1956) proposed a simple transformation of an absolutely continuous $p$-variate distribution into the uniform distribution on the $p$-dimensional hypercube and suggested using this transformation to build multivariate goodness of fit tests. The distribution function of the Cramer-von Mises statistic in the multivariate case has been studied by a number of authors (see Kotz and Johnson, 1985, pp: 35-39) but a general multivariate test of goodness of fit based on this statistic that can be readily applied has not yet been developed.

Mardia (1970) seems to be the first to have published a practical multivariate test of goodness of fit for the multinormal distribution using multivariate measures of skewness and kurtosis. Malkovich and Afifi (1973) used the univariate normality of all linear combinations to derive three tests of normality. These tests search for the linear combination with the largest measures of skewness, kurtosis and the negative of the Shapiro and Wilk statistics, respectively. Cox and Small (1978) suggested finding the linear combination of the variables maximizing the curvature when regressed on the other. Other procedures to check for multivariate normality have been proposed by Andrews et al. (1973), Dahiya and 
Gurland (1973), Hensler et al. (1977), C'sörgõ (1986), Mudholkar et al. (1992) and Ghosh and Ruymgaart (1992).

In this paper we present two multivariate goodness of fit test. In section 2 we present a multivariate goodness of fit statistics which is distribution free and reduces to the KolmogorovSmirnov statistic in the univariate case. The computation of the proposed statistic is a problem in itself, and in this section we develop another statistic that can be easily computed for any dimension. Section 3 presents a procedure to compute the test statistics in the bivariate case. In section 4 we present some simulation results for the exact and the approximated Kolmogorov-Smirnov statistics. Finally, section 5 includes some concluding remarks.

\section{The Multivariate Kolmogorov-Smirnov Statistic;}

Given a sample $x_{1}, \ldots, x_{n}$ of i.i.d. random variables with distribution function $F$, consider the problem of testing $H_{0}: F=F_{0}$ versus $H_{1}: F \neq F_{0}$, where $F_{0}$ is some specified distribution function. In the univariate case, $H_{0}$ can be tested using the Kolmogorov-Smirnov statistic.

$$
D_{n}=\sup _{x \in \mathbb{R}}\left|F_{n}(x)-F(x)\right|,
$$

where $F_{n}$ is the empirical distribution function of the sample. It is also well known that this statistic is distribution free and it can be expressed as

$$
D_{n}=\sup _{0 \leq u \leq 1}\left|G_{n}(u)-u\right|
$$

where $G_{n}(u)$ is the empirical distribution function of the uniform 0-1 transformed sample $u_{i}=F_{0}\left(y_{i}\right)$, for $i=1, \ldots, n$.

The distribution free property of the Kolmogorov-Smirnov statistic is derived from the result that any continuous random variable $X$ with distribution function $F$ can be transformed to a uniform random variable $Y$ by the transformation $Y=F(X)$. A similar result holds for a continuous multivariate random variable $\boldsymbol{X}$, as it is shown in the following theorem, due to Rosenblatt (1952).

TheOREM 1. Let $\boldsymbol{X}=\left(X_{1}, \ldots, X_{p}\right)$ be a random vector with joint density

$$
f\left(x_{1}, \ldots, x_{p}\right)=f_{1}\left(x_{1}\right) f_{2}\left(x_{2} \mid x_{1}\right) \cdots f_{p}\left(x_{p} \mid x_{1}, \ldots, x_{p-1}\right)
$$


and define the transformation $\boldsymbol{Y}=T(\boldsymbol{X})$, by

$$
\begin{aligned}
& Y_{1}=F_{1}\left(X_{1}\right) \\
& Y_{i}=F_{i}\left(X_{i} \mid X_{1}, \ldots, X_{i-1}\right) \quad i=2, \ldots, p
\end{aligned}
$$

Then $Y_{1}, \ldots, Y_{p}^{\prime}$ are i.i.d. uniform $0-1$

The probability distribution function of the statistic

$$
\sup _{\boldsymbol{x} \in \mathbf{R}^{p}}\left|F\left(x_{1}, \ldots, x_{p}\right)-F_{n}\left(x_{1}, \ldots, x_{p}\right)\right|
$$

where $F_{n}$ is the empirical distribution function, is not distribution free. However, as suggested by Rosenblatt (1952) we could use the transformation defined in theorem 1 to test whether the values $\left(y_{1}, \ldots y_{n}\right)$ are a sample from a uniform distribution on the $p$-dimensional hypercube. The natural extension of the statistic (2.1) to the multivariate case is

$$
D_{n}=\sup _{\boldsymbol{y}}\left|G_{n}(\boldsymbol{y})-y_{1} \cdots y_{p}\right|
$$

where $G_{n}$ is the empirical distribution function of the transformed sample $\boldsymbol{y}=T(\boldsymbol{x})$.

Unfortunately, and unlike in the univariate case, the computation of (2.3) is very involved, as it is shown in the next section, in which we present an algorithm for the case $p=2$. Although this algorithm could be extended to the $p>2$ case, the computation difficulties appear to be considerable. A much simpler to compute statistic, $\tilde{D}_{n}$, can be defined by taking the supremum on the set of transformed sample points $A$,

$$
\tilde{D}_{n}=\sup _{\boldsymbol{y} \in A}\left|G_{n}(\boldsymbol{y})-y_{1} \cdots y_{p}\right|
$$

which, by the same argument above, is also distribution free. When $n$ is large, $\tilde{D}_{n}$ will be close to $D_{n}$, as it is shown in the simulation results reported in Section 4 .

By theorem 1, the multivariate Kolmogorov-Smirnov statistic $D_{n}$ and its approximation $\tilde{D}_{n}$ are distribution free in the class of continuous multivariate distributions. The percentiles of the distribution of $D_{n}$ can be computed for the bivariate case by Monte Carlo simulation and table 1 presents the percentiles of this statistic in the standard case in which $F_{0}$ is completely specified by $H_{0}$. Table 2 presents the percentiles in the particular case of testing 
normality and when the parameter are estimated by the sample mean and the sample covariance matrix, that is the multivariate generalization of the Kolmogorov-Smirnov-Lilliefords statistic.

(Table 1 and 2 around here)

Table 3 presents the percentiles for the distribution of the approximated KolmogorovSmirnov statistic in the bivariate case.

(Table 3 around here)

\section{An Algorithm to compute the Bivariate Kolmogorov-Smirnov Statistic:}

In a one-dimensional sample the empirical distribution changes only in the observed points, and the univariate Kolmogorov-Smirnov statistic is obtained by evaluating the distance between the empirical and theoretical distribution functions in these points. Nevertheless, when the dimension $p$ is larger than one, the empirical distribution function jumps on an infinite number of points. Here we develop a procedure for calculating the KolmogorovSmirnov statistic (2.3) in the two-dimensional case by evaluating it on a finite set.

Since theorem 1 bolds we may assume that $u_{1}=\left(x_{1}, y_{1}\right), \ldots, u_{n}=\left(x_{n}, y_{n}\right)$ is a random sample from two independent uniform $0-1$ distributions. In this context, the pair $\left(x_{j}, y_{i}\right)$ is called an intersection point if $x_{i}<x_{j}$ and $y_{i}>y_{j}$. For $u=(x, y)$ we define the supcrior distance $D_{n}^{+}(u)=\left(G_{n}(u)-G(u)\right)$ and the inferior distance $D_{n}^{-}(u)=\left(G(u)-G_{n}(u)\right)$, where $G$ is the distribution function of two independent uniform random variables on $(0,1)$ and $G_{n}$ is the empirical distribution function. Also, the left empirical distribution function in $u$ is defined as $C_{n}\left(u^{-}\right)=\lim _{\epsilon \rightarrow 0}\left(x_{n}(x-\epsilon, y-\epsilon)\right.$. The proof is based on the behavior of the lateral Kolmogorov-Smirnov statistics $D_{n}^{+}=\sup _{u} D_{n}^{+}(u)$ and $D_{n}^{-}=\sup _{u} D_{n}^{-}(u)$.

LEMMA 1. If $x_{0}=y_{0}=0$, then $D_{n}^{+}=\max _{v \in I} D_{n}^{+}(v)$, where.

$$
I=\left\{\left(x_{j}, y_{i}\right) \mid x_{i} \leq x_{j}, y_{i} \geq y_{j} ; i, j=0,1, \ldots, n\right\}
$$

Elements in the set I are: the pair $(0,0)$, the observed points and the intersection points. 
Proof. For each $u=(x, y)$ in the unit square, let $x^{u}$ and $y^{u}$ be

$$
\begin{aligned}
& x^{u}=x_{k_{u}}=\max _{i=0,1, \ldots, n}\left\{x_{i} \mid x_{i} \leq x, y_{i} \leq y\right\} \\
& y^{u}=y_{p_{u}}=\max _{i=0,1, \ldots, n}\left\{y_{i} \mid y_{i} \leq y, x_{i} \leq x\right\} .
\end{aligned}
$$

The relationship between the coordinates is given by $x_{p_{u}} \leq \max \left\{x_{i} \leq x \mid y_{i} \leq y\right\}=x_{k_{u}}$ and $y_{k_{u}} \leq \max \left\{y_{i} \leq y \mid x_{i} \leq x\right\}=y_{p_{u}}$. Hence $\left(x^{u}, y^{u}\right) \in I$. By the definition of $\left(x^{u}, y^{u}\right)$, it is immediate that $G_{n}(x, y)=G_{n}\left(x^{u}, y\right)=G_{n}\left(x, y^{u}\right)$ and the set $\left\{x_{i} \mid x_{i} \in\left(x^{u}, x\right], y_{i} \in\left(y^{u}, y\right]\right\}$ is empty. Then

$$
G_{n}\left(x^{u}, y^{u}\right)=G_{n}\left(x^{u}, y\right)+G_{n}\left(x, y^{u}\right)-G_{n}(x, y)=G_{n}(x, y)
$$

and, therefore, for each $u$

$$
D_{n}^{+}(u)=G(x, y)-G(x, y) \leq G_{n}\left(x^{u}, y^{u}\right)-G\left(x^{u}, y^{u}\right) \leq \max _{v \in I} D_{n}^{+}(v) .
$$

Hence the lemma follows.

LEMMA 2. If $x_{0}=0, y_{0}=1, x_{n+1}=1$ and $y_{n+1}=0$, then $D_{n}^{-}=\max _{v \in P}\left(G(v)-G_{n}\left(v^{-}\right)\right)$, where.

$$
P=\left\{\left(x_{j}, y_{i}\right) \mid x_{j}>x_{i}, y_{j}<y_{i} ; i, j=0,1, \ldots, n+1\right\} .
$$

Elements in set $P$ are: the pair $(1,1)$, the intersection points and the projections of the obscrved points on the right and on the top unit square borders.

Proof. For eacl $u=(x, y)$ in the unit square, let $x^{u}$ and $y^{u}$ be

$$
\begin{aligned}
& x^{u}=x_{k_{u}}=\min _{i=0,1, \ldots, n+1}\left\{x_{i} \mid x_{i}>x\right\} \\
& y^{u}=y_{p_{u}}=\min _{i=0,1, \ldots, n+1}\left\{y_{i} \mid y_{i}>y, x_{i} \leq x\right\} .
\end{aligned}
$$

Obviously, $x_{k_{u}}>x \geq x_{p_{u}}$ and $y_{p_{u}}>y$. Hence $\left(x^{u}, y^{u}\right)$ is in the set

$$
\begin{aligned}
Q & =\left\{\left(x_{j}, y_{i}\right) \mid x_{j}>x_{i} ; i, j=0,1, \ldots, n+1\right\} \\
& =P \cup\left\{\left(x_{j}, y_{i}\right) \mid x_{j}>x_{i}, y_{j}>y_{i} ; i, j=1, \ldots, n\right\} .
\end{aligned}
$$

Since $F$ is continuous and increasing, the inferior distance is bounded by

$$
\begin{aligned}
D_{n}^{-}(u) & =G(u)-G_{n}(u)<G\left(x^{u}, y^{u}\right)-G_{n}(u)= \\
& =G\left(x^{u}, y^{u}\right)-\lim _{\epsilon \rightarrow 0} G_{n}^{v}\left(x^{u}-\epsilon, y^{u}-\epsilon\right)+\lim _{\epsilon \rightarrow 0} G_{n}\left(x^{u}-\epsilon, y^{u}-\epsilon\right)-G_{n}(u) \leq \\
& \leq \max _{v \in Q}\left(G(v)-G_{n}\left(v^{-}\right)\right)+\lim _{\epsilon \rightarrow 0} G_{n}\left(x^{u}-\epsilon, y^{u}-\epsilon\right)-G_{n}(u) .
\end{aligned}
$$


Because of the definition of $\left(x^{u}, y^{u}\right), G_{n}(u)=\lim _{\epsilon \rightarrow 0} G_{n}\left(x^{u}-\epsilon, y\right)=\lim _{c \rightarrow 0} G_{n}\left(x, y^{u}-\epsilon\right)$ and the set $\left\{\left(x_{i}, y_{i}\right) \mid x_{i} \in\left(x, x^{u}\right), y_{i} \in\left(y, y^{u}\right)\right\}$ is empty. The left empirical distribution function verifies

$$
\lim _{\imath \rightarrow 0} G_{n}\left(x^{u}-\epsilon, y^{u}-\epsilon\right)=\lim _{\epsilon \rightarrow 0} G_{n}\left(x^{u}, y-\epsilon\right)+\lim _{\epsilon \rightarrow 0} G_{n}\left(x, y^{u}-\epsilon\right)-G_{n}(u)=G_{n}(u)
$$

In addition, if $\left(x^{u}, y^{u}\right) \in Q-P$, we define $x^{w}=\min \left\{x_{i} \mid x_{i}>x^{u}, y_{i}<y^{u}\right\}$. Then the pair $\left(x^{w}, y^{u}\right)$ is in $P, G\left(x^{u}, y^{u}\right)<G\left(x^{w}, y^{u}\right)$ and the set $\left\{\left(x_{i}, y_{i}\right) \mid x_{i} \in\left[x^{u}, x^{w}\right), y_{i} \in\left(-\infty, y^{u}\right)\right\}$ is empty. Hence $\lim _{\epsilon \rightarrow 0} G_{n}\left(x^{w}-\epsilon, y^{u}-\epsilon\right)=\lim _{c \rightarrow 0} G_{n}\left(x^{u}-\epsilon, y^{u}-\epsilon\right)$ and

$$
G\left(x^{u}, y^{u}\right)-\lim _{\epsilon \rightarrow 0} G_{n}\left(x^{u}-\epsilon, y^{u}-\epsilon\right)<\max _{v \in P}\left(G(v)-G_{n}\left(v^{-}\right)\right) \text {. }
$$

By (3.4), (3.5) and (3.6), $\max _{v \in P}\left(G(v)-G_{n}\left(v^{-}\right)\right)$is a superior bound for $D_{n}^{-}(u)$.

Finally, let $u_{0}=\left(x_{0}, y_{0}\right)$ be given by $u_{0}=\arg \max _{v \in P}\left(G(v)-G_{n}\left(v^{-}\right)\right)$, then

$$
\begin{aligned}
\max _{v \in P}\left(G(v)-G_{n}\left(v^{-}\right)\right) & =\lim _{i \rightarrow 0}\left(G\left(x_{0}-\epsilon, y_{0}-\epsilon\right)-\left(x_{n}\left(x_{0}-\epsilon, y_{0}-\epsilon\right)\right)=\right. \\
& =\lim _{\epsilon \rightarrow 0} D_{n}^{-}\left(x_{0}-\epsilon, y_{0}-\epsilon\right) .
\end{aligned}
$$

Hence $D_{n}^{-}=\max _{v \in P}\left(G^{\prime}(v)-G_{n}\left(v^{-}\right)\right)=\sup _{u} D_{n}^{-}(u)$ and the lemma follows.

THEOREM 2. If $p=2$, the Kolmogorov-Smirnov statistic (2.3) is

$$
D_{n}=\max _{u \in J, v \in P}\left\{G_{n}(u)-G(u), G(v)-G_{n}\left(v^{-}\right)\right\}
$$

The Kolmogorov-Smirnov statistics may be expressed as $D_{n}=\max \left\{D_{n}^{+}, D_{n}^{-}\right\}$and the proof is straightforward by lemmas 1 and 2 .

As a consequence of theorem 2, $D_{n}$ may be obtained by evaluating the distance in a finite amount of points which ranks from $3 n$ to $3 n+\left(\begin{array}{l}n \\ 2\end{array}\right)$ depending on the sample configuration. The theorem leads to the following procedure to compute the Kolmogorov-Smirnov statistic (2.3):

1. Compute the maximum distance in the observed points, $D_{n}^{1}=\max _{i=1, \ldots, n} D_{n}^{+}\left(u_{i}\right)$.

2. Compute the maximum and minimum distances in the intersection points, $D_{n}^{2}=\max _{i, j=1, \ldots, n}\left\{D_{n}^{+}\left(x_{j}, y_{i}\right) \mid x_{j}>x_{i}, y_{j}<y_{i}\right\}$ and $D_{n}^{3}=2 / n-\min _{i, j=1, \ldots, n}\left\{D_{n}^{+}\left(x_{j}, y_{i}\right)\right.$ $\left.x_{j}>x_{i}, y_{j}<y_{i}\right\}$. 
3. Compute the maximum distance among the projections of the observed points on the right unit square border, $D_{n}^{4}=1 / n-\min _{i=1, \ldots, n} D_{n}^{+}\left(1, y_{i}\right)$.

4. Compute the maximum distance among the projections of the observed points on the top unit square border, $D_{n}^{5}=1 / n-\min _{i=1, \ldots, n} D_{n}^{+}\left(x_{i}, 1\right)$.

5. Compute the maximum $D_{n}=\max \left\{D_{n}^{1}, D_{n}^{2}, D_{n}^{3}, D_{n}^{4}, D_{n}^{5}\right\}$.

\section{Some Simulation Results}

The power of the exact and the approximate multivariate Kolmogorov-Smirnov statistics when used as a normality test and as a general multivariate goodness of fit test have been investigated. In the first case, the null hypothesis is bivariate normal with mean $\boldsymbol{\mu}=0$ and covariance matrix

$$
\boldsymbol{\Sigma}=\left(\begin{array}{cc}
1 & 0.5 \\
0.5 & 1
\end{array}\right)
$$

The alternative distribution is

$$
(1-\epsilon) N(\mathbf{0}, \boldsymbol{\Sigma})+\epsilon N(\boldsymbol{\mu}, \boldsymbol{\Sigma})
$$

for several values of $\epsilon$ and $\boldsymbol{\mu}$. Tabla 4 shows the power of the normality test. As we may have expected, the power increases with $n$ and is larger for the exact test than for the approximate one. However, for moderately large $n$ ( $n \geq 50$ say) the power of the approximate test is very close to that of the exact one. Table 4 shows that both tests are very powerful when $n$ is large and $\epsilon \geq 0.2$.

(Table 4 around liere)

Table 5 shows the power of these statistics when the null distribution is Morgenstern (see Morgenstern, 1956) with parameters $\alpha=0.5$. Similar results were also found for other values of $\alpha$. We have chosen this distribution because it may have fixed marginal distribution allowing different degrees of dependency. The joint density function for the uniform marginal case is

$$
f\left(x_{1}, x_{2}\right)=1+\alpha\left(2 x_{1}-1\right)\left(2 x_{2}-1\right) \quad 0 \leq x_{1}, x_{2} \leq 1 \quad-1 \leq \alpha \leq 1
$$


and it is straightforward to show that for this distribution

$$
\begin{gathered}
F\left(x_{1}\right)=x_{1} \quad 0 \leq x_{1} \leq 1 \\
F\left(x_{2} \mid x_{1}\right)=\left(1-\alpha\left(2 x_{1}-1\right)\right) x_{2}+\alpha\left(2 x_{1}-1\right) x_{2}^{2} \quad 0 \leq x_{2} \leq 1 .
\end{gathered}
$$

The alternative distributions are independent Beta distributions with several combination of shape parameters, to allow for different degrees of asymmetry. Table 5 shows that again, as one could expect, for $n$ small $(n=10)$ the power is very low unless the degree of kurtosis or asymmetry is high. The difference between the power of the exact and approximate test is negligible for large $n(n \leq 50)$.

(Table 5 around here)

\section{CONCLUDING REMARlis}

As in the univariate case, the multivariate Kolmogorov-Smirnov test presented in this paper may provide a general and flexible goodness of fit test, specially for situations when specific test are yet to be developed. The main problem in the application of the test is the computation of the statistic in the case $p>2$. An extension of the computing algorithm developed in this paper may be possible, but still the numerical complications seem considerable. However, our simulation results show that the approximate KolmogorovSmirnov test statistics introduced in this paper, that is trivial to compute, seems to be a promising alternative with a very small loss of power when $n$ is moderately large.

\section{ACKNOWLEDGEMENTS}

We are very grateful to Victor Yohai for useful discussion and comments. A. Justel and D. Peña were partly supported by PB90-0266 grant from DGICYT (Spain) and R. Zamar was partly supported by a grant from National Science and Engineering Research Council of Canada (NSERC). 


\section{REFERENCES}

Andrews, D.F., Gnanadesikan, R. \& Warner, J.L. (1973). Methods for assessing multivariate normality. In Multivariate Analysis, Vol. 3, Ed. P.R. Krishnaiah, pp. 95-116. New York: Academic Press.

Cox, D.R. \& Small, N.J.H. (1978). Testing multivariate normality. Biometrika, 65, 263272.

Csörgõ, S. (1986). Testing for normality in arbitrary dimension. Ann. Statist., 14, 708-723.

D'Agostino, R.B. \& Stephend, M.A. (1986). Goodness-of-fit Techniques. New York: Marcel Dekker.

DahiYa, R.C. \& Gurland, J. (1973). A test of fit for bivariate distributions. J. R. Statist. Soc. B, 35, 452-465.

Ghosh, S. \& RUYmgaART, F.H. (1992). Applications of empirical characteristic functions in some multivariate problems. Can. J. Statist., 20, 429-440.

Hensler, G.L., Mehrotra, K.G. \& Michalek, J.E. (1977). A Goodness of fit test for multivariate normality. Comm. Statist. Theor. Meth. A, 6, 33-41.

Kotz, S. \& Johnson, N.L. (1983). Encyclopedia of Statistical Sciences. New York: John Wiley.

Kotz, S. \& Johnson, N.L. (1985). Encyclopedia of Statistical Sciences, Vol. 6. New York: John Wiley.

Krishnalah, P.R. (1980). Handbook of Statistics. North-Holland.

Malkovich, J.F. \& AfIFı, A.A. (1973). On tests for multivariate normality. J. Am. Statist. Assoc., 68, 176-179.

MARDIA, K.V. (1970). Measures of multivariate skewness and kurtosis with applications. Biometrika, 57, 519-530.

Moore, D.S. \& Stubblebine, J.B. (1981). Chi-square tests for multivariate normality, with application to common stock prices. Comm. Statist. Theor. Meth. A, 10, 713-733.

Morgenstern, D. (1956). Einfacle beispiele zweidimensionaler verteilungen. Mitt. Math. Statistik, 8, 234-235.

Mudholkar, G.S., McDermott, M. \& Srivastava, D.K. (1992). A test of p-variate normality. Biometrika, 79, 4, 850-854. 
RaO, K.C. \& Robson, D.S. (1974). A chi-square statistics for goodness-of-fit within the exponential family. Comm. Statist., 3, 1139-1153.

Rosenblatt, M. (1952). Remarks on a multivariate transformation. Ann. Math. Statist., 23, 470-472. 
Table 1: Monte Carlo approximation to the percentiles of the Kolmogorov-Smirnov statistic distribution, with 2,000 replications.

$\begin{array}{rcccccc}\mathrm{n} & 0.25 & 0.2 & 0.15 & 0.1 & 0.05 & 0.01 \\ 10 & 0.4076 & 0.4244 & 0.4432 & 0.4668 & 0.5022 & 0.5731 \\ 11 & 0.3863 & 0.4022 & 0.4190 & 0.4408 & 0.4796 & 0.5548 \\ 12 & 0.3739 & 0.3882 & 0.4041 & 0.4275 & 0.4629 & 0.5229 \\ 13 & 0.3576 & 0.3716 & 0.3883 & 0.4082 & 0.4378 & 0.5015 \\ 14 & 0.3491 & 0.3623 & 0.3753 & 0.3988 & 0.4246 & 0.4874 \\ 15 & 0.3383 & 0.3516 & 0.3644 & 0.3871 & 0.4116 & 0.4657 \\ 16 & 0.3222 & 0.3347 & 0.3517 & 0.3699 & 0.4018 & 0.4637 \\ 17 & 0.3177 & 0.3291 & 0.3435 & 0.3628 & 0.3896 & 0.4415 \\ 18 & 0.3120 & 0.3216 & 0.3349 & 0.3526 & 0.3819 & 0.4321 \\ 19 & 0.3016 & 0.3137 & 0.3271 & 0.3435 & 0.3718 & 0.4237 \\ 20 & 0.2923 & 0.3041 & 0.3161 & 0.3329 & 0.3585 & 0.4088 \\ 25 & 0.2643 & 0.2733 & 0.2849 & 0.3019 & 0.3251 & 0.3759 \\ 30 & 0.2410 & 0.2498 & 0.2602 & 0.2744 & 0.2964 & 0.3501 \\ 35 & 0.2238 & 0.2330 & 0.2441 & 0.2601 & 0.2831 & 0.3221 \\ 50 & 0.1901 & 0.1981 & 0.2063 & 0.2174 & 0.2330 & 0.2763 \\ 100 & 0.1360 & 0.1414 & 0.1473 & 0.1559 & 0.1686 & 0.1934\end{array}$


Table 2: Monte Carlo approximation to the Kolmogorov-Smirnov Lilliefords statistic distribution, with 2,000 replications.

$\begin{array}{rcccccc}\mathrm{n} & 0.25 & 0.2 & 0.15 & 0.1 & 0.05 & 0.01 \\ 10 & 0.3067 & 0.3155 & 0.3264 & 0.3416 & 0.3609 & 0.4040 \\ 11 & 0.2935 & 0.3031 & 0.3140 & 0.3285 & 0.3539 & 0.3895 \\ 12 & 0.2818 & 0.2892 & 0.2990 & 0.3096 & 0.3274 & 0.3725 \\ 13 & 0.2713 & 0.2796 & 0.2889 & 0.3004 & 0.3227 & 0.3650 \\ 14 & 0.2635 & 0.2713 & 0.2803 & 0.2946 & 0.3110 & 0.3473 \\ 15 & 0.2583 & 0.2654 & 0.2749 & 0.2870 & 0.2997 & 0.3333 \\ 16 & 0.2489 & 0.2561 & 0.2646 & 0.2760 & 0.2922 & 0.3338 \\ 17 & 0.2400 & 0.2477 & 0.2567 & 0.2685 & 0.2888 & 0.3175 \\ 18 & 0.2384 & 0.2455 & 0.2536 & 0.2637 & 0.2805 & 0.3077 \\ 19 & 0.2307 & 0.2379 & 0.2467 & 0.2579 & 0.2759 & 0.3063 \\ 20 & 0.2253 & 0.2318 & 0.2410 & 0.2504 & 0.2673 & 0.3022 \\ 25 & 0.2026 & 0.2088 & 0.2163 & 0.2254 & 0.2407 & 0.2773 \\ 30 & 0.1862 & 0.1916 & 0.1981 & 0.2080 & 0.2204 & 0.2490 \\ 35 & 0.1737 & 0.1798 & 0.1862 & 0.1932 & 0.2069 & 0.2325 \\ 50 & 0.1469 & 0.1508 & 0.1556 & 0.1625 & 0.1737 & 0.1910 \\ 100 & 0.1020 & 0.1048 & 0.1081 & 0.1125 & 0.1187 & 0.1329\end{array}$


Table 3: Monte Carlo approximation to the percentiles of the approximated KolmogorovSmirnov statistic distribution, with 2,000 replications.

$\begin{array}{rcccccc}\mathrm{n} & 0.25 & 0.2 & 0.15 & 0.1 & 0.05 & 0.01 \\ 10 & 0.3044 & 0.3209 & 0.3386 & 0.3654 & 0.4056 & 0.4758 \\ 11 & 0.2914 & 0.3085 & 0.3264 & 0.3511 & 0.3896 & 0.4568 \\ 12 & 0.2854 & 0.2998 & 0.3176 & 0.3452 & 0.3785 & 0.4394 \\ 13 & 0.2732 & 0.2874 & 0.3066 & 0.3266 & 0.3589 & 0.4278 \\ 14 & 0.2641 & 0.2770 & 0.2903 & 0.3117 & 0.3440 & 0.4194 \\ 15 & 0.2631 & 0.2764 & 0.2907 & 0.3105 & 0.3494 & 0.4221 \\ 16 & 0.2483 & 0.2640 & 0.2772 & 0.2970 & 0.3266 & 0.3838 \\ 17 & 0.2441 & 0.2572 & 0.2748 & 0.2964 & 0.3277 & 0.3875 \\ 18 & 0.2377 & 0.2484 & 0.2613 & 0.2812 & 0.3083 & 0.3690 \\ 19 & 0.2351 & 0.2455 & 0.2569 & 0.2764 & 0.3058 & 0.3631 \\ 20 & 0.2303 & 0.2401 & 0.2505 & 0.2702 & 0.2978 & 0.3510 \\ 25 & 0.2072 & 0.2168 & 0.2284 & 0.2438 & 0.2715 & 0.3261 \\ 30 & 0.1919 & 0.2026 & 0.2138 & 0.2303 & 0.2513 & 0.2878 \\ 35 & 0.1798 & 0.1883 & 0.1992 & 0.2147 & 0.2365 & 0.2786 \\ 50 & 0.1511 & 0.1586 & 0.1670 & 0.1797 & 0.1993 & 0.2396 \\ 100 & 0.1134 & 0.1180 & 0.1249 & 0.1325 & 0.1449 & 0.1708\end{array}$


Table 4: Empirical power of the Kolmogorov-Smirnov (KS) and approximated KolmogorovSmirnov (AKS) test with size 0.1. The null hypothesis is a $N(0, \Sigma)$ and the samples are generated from a normal mixture $(1-\epsilon) N(0, \Sigma)+\epsilon N(\mu, \Sigma)$.

\begin{tabular}{|c|c|c|c|c|c|c|c|c|c|}
\hline & & \multicolumn{2}{|c|}{$n=15$} & \multicolumn{2}{|c|}{$n=25$} & \multicolumn{2}{|c|}{$n=50$} & \multicolumn{2}{|c|}{$n=100$} \\
\hline & & $\mathrm{KS}$ & AKS & $\mathrm{KS}$ & $\mathrm{AKS}$ & $\mathrm{KS}$ & $\mathrm{AKS}$ & $\mathrm{KS}$ & $\mathrm{AKS}$ \\
\hline \multirow[t]{3}{*}{$\boldsymbol{\mu}=(3,3)^{\prime}$} & $\epsilon=0.1$ & 0.15 & 0.13 & 0.18 & 0.16 & 0.27 & 0.25 & 0.44 & 0.41 \\
\hline & $\epsilon=0.2$ & 0.30 & 0.26 & 0.45 & 0.41 & 0.72 & 0.70 & 0.96 & 0.95 \\
\hline & $\epsilon=0.4$ & 0.75 & 0.73 & 0.93 & 0.92 & 1.00 & 1.00 & 1.00 & 1.00 \\
\hline \multirow[t]{3}{*}{$\boldsymbol{\mu}=(3,-1)^{\prime}$} & $\epsilon=0.1$ & 0.16 & 0.11 & 0.21 & 0.14 & 0.30 & 0.21 & 0.50 & 0.36 \\
\hline & $\epsilon=0.2$ & 0.34 & 0.20 & 0.51 & 0.33 & 0.77 & 0.60 & 0.97 & 0.91 \\
\hline & $\epsilon=0.4$ & 0.80 & 0.59 & 0.95 & 0.83 & 1.00 & 0.99 & 1.00 & 1.00 \\
\hline
\end{tabular}


Table 5: Empirical power of the Kolmogorov-Smirnov (KS) and the approximated Kolmogorov-Smirnov (AKS) test of size $\alpha$. The null hypethesis is a Morgenstern with parameter 0.5 and uniform marginals. The samples are generated from two independent Beta $(a, b)$.

\begin{tabular}{|c|c|c|c|c|c|c|c|}
\hline & \multirow[b]{2}{*}{$\mathbf{n}$} & \multicolumn{2}{|c|}{$\alpha=0.1$} & \multicolumn{2}{|c|}{$\alpha=0.05$} & \multicolumn{2}{|c|}{$\alpha=0.01$} \\
\hline & & $\mathrm{KS}$ & AKS & $\mathrm{KS}$ & AKS & $\mathrm{KS}$ & AKS \\
\hline \multirow[t]{4}{*}{$\operatorname{Beta}(10,10)$} & 10 & 0.948 & 0.424 & 0.842 & 0.310 & 0.372 & 0.157 \\
\hline & 20 & 1.000 & 0.777 & 0.999 & 0.692 & 0.994 & 0.505 \\
\hline & 50 & 1.000 & 0.999 & 1.000 & 0.996 & 1.000 & 0.975 \\
\hline & 100 & 1.000 & 1.000 & 1.000 & 1.000 & 1.000 & 1.000 \\
\hline \multirow[t]{4}{*}{ Beta $(3,3)$} & 10 & 0.241 & 0.177 & 0.129 & 0.101 & 0.020 & 0.029 \\
\hline & 20 & 0.640 & 0.385 & 0.459 & 0.260 & 0.175 & 0.097 \\
\hline & 50 & 0.992 & 0.897 & 0.968 & 0.793 & 0.756 & 0.502 \\
\hline & 100 & 1.000 & 0.999 & 1.000 & 0.997 & 0.999 & 0.973 \\
\hline \multirow[t]{4}{*}{$\operatorname{Beta}(3,2)$} & 10 & 0.167 & 0.145 & 0.079 & 0.059 & 0.008 & 0.007 \\
\hline & 20 & 0.518 & 0.424 & 0.332 & 0.244 & 0.089 & 0.053 \\
\hline & 50 & 0.983 & 0.969 & 0.943 & 0.878 & 0.574 & 0.471 \\
\hline & 100 & 1.000 & 1.000 & 1.000 & 1.000 & 0.998 & 0.992 \\
\hline \multirow[t]{4}{*}{$\operatorname{Beta}(0.5,1)$} & 10 & 0.579 & 0.575 & 0.439 & 0.422 & 0.220 & 0.211 \\
\hline & 20 & 0.842 & 0.816 & 0.753 & 0.713 & 0.508 & 0.451 \\
\hline & 50 & 0.994 & 0.993 & 0.989 & 0.98 .5 & 0.911 & 0.898 \\
\hline & 100 & 1.000 & 1.000 & 1.000 & 1.000 & 0.999 & 0.999 \\
\hline \multirow[t]{4}{*}{$\operatorname{Beta}(0.5,0.5)$} & 10 & 0.308 & 0.247 & 0.212 & 0.153 & 0.077 & 0.053 \\
\hline & 20 & 0.516 & 0.403 & 0.388 & 0.284 & 0.186 & 0.111 \\
\hline & 50 & 0.850 & 0.792 & 0.759 & 0.651 & 0.456 & 0.358 \\
\hline & 100 & 0.994 & 0.987 & 0.976 & 0.960 & 0.887 & 0.832 \\
\hline
\end{tabular}

